\title{
Bonding Trends and Dimensionality Crossover of Gold Nanoclusters on Metal-Supported MgO Thin Films
}

\author{
Davide Ricci, ${ }^{1}$ Angelo Bongiorno, ${ }^{1}$ Gianfranco Pacchioni, ${ }^{2}$ and Uzi Landman ${ }^{1}$ \\ ${ }^{1}$ Georgia Institute of Technology, School of Physics, Atlanta, Georgia 30332-0430, USA \\ ${ }^{2}$ Dipartimento di Scienza dei Materiali, Università Milano-Bicocca, via Cozzi, 53-20125 Milano, Italy
} (Received 28 April 2006; published 21 July 2006)

Bonding of gold clusters, $\mathrm{Au}_{n} n=8,16$, and 20, on $\mathrm{MgO}(100)$ and on thin $\mathrm{MgO}$ films supported on $\mathrm{Mo}(100)$ is investigated using first-principles density-functional theory. Enhanced adhesive bonding is found for clusters deposited on metal-supported $\mathrm{MgO}$ films of thickness up to about $1 \mathrm{~nm}$, or 4 to $5 \mathrm{MgO}$ layers, originating from electrostatic interaction between the underlying metal and metal-induced excess electronic charge accumulated at the cluster interface with the oxide film. The increased wetting propensity is accompanied by a dimensionality crossover from three-dimensional optimal cluster geometries on $\mathrm{MgO}(100)$ to energetically favored two-dimensional structures on the metal-supported films.

DOI: 10.1103/PhysRevLett.97.036106

PACS numbers: 68.47.Jn, 68.35.-p

Materials clusters of nanoscale sizes often exhibit structural, electronic, spectral, magnetic, and chemical characteristics that are unique to this size regime and which cannot be extrapolated or deduced through scaling arguments from knowledge of these properties in the bulk limit [1]. While nanoclusters are commonly investigated in the gas phase, there is a continuing growing interest in their properties when deposited on solid substrates. We note here the recent surge in research pertaining to the catalytic activity of gold nanoclusters supported on metal-oxide (MO) surfaces [2], where cluster morphology, electronic structure, and charge state, as well as the state of the MO surface (defect-rich containing oxygen-vacancy $F$ centers, or defect poor), have been found to govern the chemical activity [3-8]. In such surface-anchored cluster systems it would be highly desirable to find ways to control and tune the properties of the adsorbed clusters through manipulations of the supporting substrate. Cluster properties that may be influenced via substrate manipulations include: adsorption (adhesive) energies, cluster geometries and dimensionalities, cluster diffusion barriers and coalescence propensity, electronic spectra, charge distributions, and chemical reactivities.

Here we report on first-principles investigations focusing on the energetics, atomic structure, dimensionality, and chemical properties of $\mathrm{Au}$ nanoclusters adsorbed on thin $\mathrm{MgO}$ films. In particular, we consider, comparatively, the $\mathrm{MgO}(100)$ crystalline surface and $\mathrm{MgO}$ thin films grown on a $\mathrm{Mo}(100)$ substrate [commonly employed in experiments pertaining to the catalytic properties of nanosized Au clusters adsorbed on the $\mathrm{MgO}$ films [5,9] ]. We find that the adsorption of $\mathrm{Au}$ clusters on $\mathrm{MgO}(100)$ surface involves accumulation of electronic charge at the clusteroxide interface. In this case, adhesion is weak and the adsorption process does not influence the energetics of the Au clusters in a noticeable manner. On the other hand, the adhesion energy of Au clusters increases most significantly on metal [i.e., $\mathrm{Mo}(100)$ ] supported $\mathrm{MgO}$ thin films. The enhanced wettability correlates with the amount of additional electronic charge accumulated at the clusteroxide interface which increases with the number of $\mathrm{Au}$ atoms in direct contact with the oxide surface. The electrostatic interaction between the charge accumulated at the cluster-oxide interface and the metal substrate underlies the gain in adhesion energy. The extra attraction of the clusters to the $\mathrm{MgO}$ surface broadens significantly the energy distribution of the cluster isomers (for a given cluster size), with those showing lower dimensionality (i.e., planar atomic arrangements) exhibiting enhanced stability. The above electrostatic stabilization effect decays with the oxide thickness and it remains appreciable up to about $1 \mathrm{~nm}$ thick $\mathrm{MgO}$ films supported on $\mathrm{Mo}(100)$.

Our first-principles calculations are based on a densityfunctional theory approach [10,11], with exchange and correlation energy corrections included through a generalized gradient approximation (GGA) [12]. A plane wave basis is used with a cutoff kinetic energy of $30 \mathrm{Ry}$, and ultrasoft pseudopotentials [13] are employed with $\Gamma$-point sampling of the Brillouin zone. In structural relaxations corresponding to minimization of the total energy, convergence is achieved when the forces on the atoms are less than $0.015 \mathrm{eV} / \AA$.

In modeling the metal-supported $\mathrm{MgO}$ films we use a four-layer $\operatorname{Mo}(100)$ slab (lattice constant of $3.15 \AA$ ) which has been found to reproduce (in its middle) the bulk electronic properties of Mo [14]. We adsorb $\mathrm{Au}_{n}$ clusters containing $n=8,16,20 \mathrm{Au}$ atoms. In the case of $\mathrm{Au}_{8}$ we use a $\mathrm{MgO} / \mathrm{Mo}(100)-(4 \times 4)$ slab while for the clusters with $n=16$ and $n=20$ we employ a $\mathrm{MgO} / \mathrm{Mo}(100)-(5 \times 5)$ slab; $(n \times n)$ denotes the number of unit cells in the Mo(100) surface. In all calculations, the periodically replicated slabs are separated [in the (100) direction] by a vacuum region of $20 \AA$. In structural optimizations all the atoms of the adsorbed gold clusters, the 
$\mathrm{MgO}$ thin film, and the first two layers of the Mo substrate are allowed to relax.

We consider first the energetics of Au nanoclusters on a $\mathrm{MgO}(100)$ crystalline surface, modeled by a two-layer $\mathrm{MgO}(100)$ slab which is sufficiently thick to both reproduce the properties of the bare $\mathrm{MgO}$ surface [15] and to obtain converged results (with respect to the number of $\mathrm{MgO}$ layers) for the energetics of adsorbed $\mathrm{Au}$ clusters [16]. To allow direct comparison with the energetics of $\mathrm{Au}$ clusters on $\mathrm{MgO}$ thin films supported on $\mathrm{Mo}(100)$ (vide infra), we use here the in-plane lattice constant required to match the $\operatorname{Mo}(100)$ surface $(5 \%$ greater than the calculated lattice constant of bulk $\mathrm{MgO}$ ). We verified that the use of the increased lattice constant does not alter substantially the energetics of the Au clusters adsorbed on the $\mathrm{MgO}$ surface. The first $\mathrm{MgO}$ layer is held static while the remaining $\mathrm{MgO}$ layer and the gold clusters are allowed to relax. We consider two isomers of $\mathrm{Au}_{8}$, four for $\mathrm{Au}_{16}$, and two for $\mathrm{Au}_{20}$ [5,17-19]. Upon adsorption the number of gold atoms in contact with the $\mathrm{MgO}$ surface $\left(n_{\text {cont }}\right)$ differs between the isomers. We calculate the adhesion energy by referring the energy of the full system $\left(\mathrm{Au}_{n} / \mathrm{MgO}\right)$ to that of the isolated $\mathrm{Au}_{n}$ isomer and the $\mathrm{MgO}$ slab, $E_{\mathrm{adh}}=-\left[E_{\left(\mathrm{Au}_{n} / \mathrm{MgO}\right)}-E_{\mathrm{Au}_{n}}-E_{\mathrm{MgO}}\right]$. To compare the energy of adsorbed cluster isomers of the same size, the energy of the most stable isomer in vacuum is used as a reference.

The calculated adhesion energy of the Au clusters, normalized to the number of atoms in the contact area $\left(E_{\text {adh }} / n_{\text {cont }} \equiv \tilde{E}\right)$, does not depend on the cluster size and it correlates well with $n_{\text {cont }}$. For exceedingly small contacts, $n_{\text {cont }}=3, \tilde{E}$ takes a value of about $0.8 \mathrm{eV}$ and then decreases rapidly for larger values of $n_{\text {cont }}$, reaching a value of about $0.2 \mathrm{eV}$ for $n_{\text {cont }}=20$ (Fig. 1). The relative stability of the adsorbed cluster isomers (ordered according to their total energies) changes only in a few instances from the vacuum case and it does not depend on $n_{\text {cont }}$. These results are a consequence of the relatively weak adhesion of nanosize Au clusters on $\mathrm{MgO}(100)$.

To explore the energetics of $\mathrm{Au}$ nanoclusters adsorbed on metal-supported $\mathrm{MgO}$ thin films we calculate the adhesion energy of the above selected isomers of gold nanoclusters deposited on a two-layer $\mathrm{MgO}$ thin film supported by $\mathrm{Mo}(100)$. Here, the adhesion energy of the Au cluster is obtained by referring the energy of the combined system, $\mathrm{Au}_{n} / \mathrm{MgO} / \mathrm{Mo}(100)$, to that of the cluster isomer in vacuum and the isolated $\mathrm{MgO} / \mathrm{Mo}(100)$ slab. The normalized adhesion energy, $\tilde{E}$, on $\mathrm{MgO} / \mathrm{Mo}(100)$ shows a dependence on the contact area at the cluster-oxide interface, similar to the aforementioned case of the adsorption on the $\mathrm{MgO}(100)$ surface (Fig. 1). However, on $\mathrm{MgO} / \mathrm{Mo}(100)$, the $\tilde{E}$ values are shifted to higher values for the full range of contact areas (Fig. 1). In particular, we find an increment of more than $0.6 \mathrm{eV}$ in the $\tilde{E}$ for $n_{\text {cont }}<4$ and a nearly uniform increase of about $0.3 \mathrm{eV}$ for $n_{\text {cont }}>7$ (Fig. 2). The stronger adhesion on $\mathrm{MgO} / \mathrm{Mo}(100)$ produces two

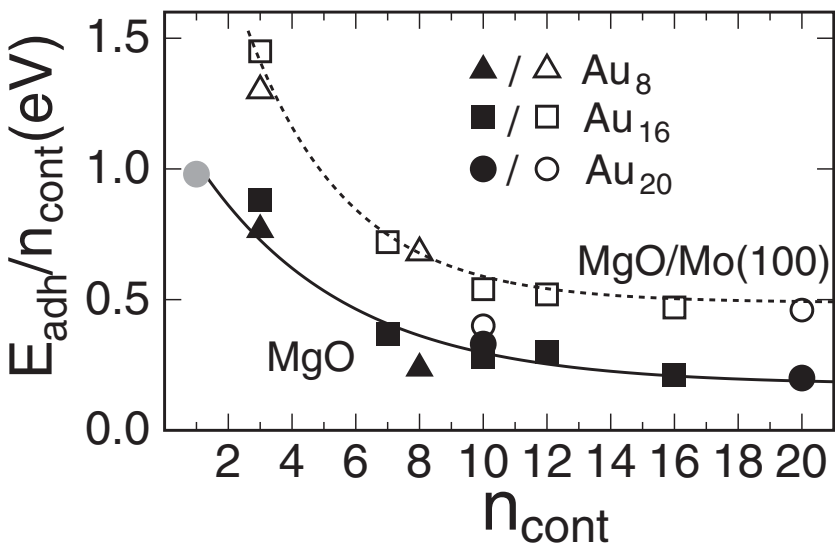

FIG. 1. Adhesion energy per contact atom, $\tilde{E}$, of $\mathrm{Au}_{n}$ cluster isomers on $\mathrm{MgO}(100)$ (filled symbols) and on a two-layer $\mathrm{MgO}$ thin film supported on $\mathrm{Mo}(100)$ (empty symbols), plotted vs the number of gold atoms in contact with the oxide surface $\left(n_{\text {cont }}\right)$. Triangles, squares, and circles denote the selected isomers for the $\mathrm{Au}_{8}, \mathrm{Au}_{16}$, and $\mathrm{Au}_{20}$ clusters, respectively. Lines are added to guide the eyes. Also included is the adhesion energy for a single $\mathrm{Au}$ atom on $\mathrm{MgO}$ (gray circle) [20]. The adhesion energy of a single $\mathrm{Au}$ atom on $\mathrm{MgO} / \mathrm{Mo}(100)$ falls on the continuation of the dashed line (not shown) [20].

marked effects on the energetics of the Au clusters: (i) the energy distributions of the isomers of the $\mathrm{Au}_{8}$, $\mathrm{Au}_{16}$, and $\mathrm{Au}_{20}$ clusters broadens with respect to the adsorption on $\mathrm{MgO}(100)$ by factors of about 3, 5, and 3, respectively, and, most interestingly, (ii) gold clusters with large contact areas with the $\mathrm{MgO}$ surface become energetically favored. In particular, planar $\mathrm{Au}_{n}$ clusters with all the $n \mathrm{Au}$ atoms in contact with the $\mathrm{MgO}$ surface, corresponding to maximal wetting of the surface, are found to be the most stable adsorbed cluster configurations on $\mathrm{MgO} / \mathrm{Mo}(100)$.

In contrast to the above preference for planar configurations on $\mathrm{MgO} / \mathrm{Mo}(100)$, the optimal geometry of a $\mathrm{Au}_{8}$ cluster adsorbed on $\mathrm{MgO}(100)$ is three-dimensional (3D) with $3 \mathrm{Au}$ atoms in the contact region [Fig. 2(a)]. This configuration is $0.3 \mathrm{eV}$ lower in energy with respect to a planar cluster isomer adsorbed in full contact (i.e., $n_{\text {cont }}=$ 8) with the $\mathrm{MgO}(100)$ surface [Fig. 2(b)]. This situation is inverted on $\mathrm{MgO} / \mathrm{Mo}(100)$. Indeed, in this case the planar shape is favored by at least $0.8 \mathrm{eV}$ with respect to other adsorbed cluster configurations with higher dimensionality. Similar effects are found for $\mathrm{Au}_{16}$ and $\mathrm{Au}_{20}$ [Fig. 2(c)2(f)]. For $\mathrm{Au}_{16}$ on $\mathrm{MgO}(100)$, the planar isomer is lower in energy with respect to layered or $3 \mathrm{D}$ isomers by about $0.5 \mathrm{eV}$, and this energy separation increases by more than $1.7 \mathrm{eV}$ on $\mathrm{MgO} / \mathrm{Mo}(100)$ in favor of the planar isomer. The tendency toward planar (2D) optimal cluster adsorption geometries culminates for $\mathrm{Au}_{20}$ where on $\mathrm{MgO}(100)$ the pyramidal adsorbed isomer remains [as in vacuum [18] ] the most stable configuration, with a $1.2 \mathrm{eV}$ advantage over the planar one [Fig. 2(e) and 2(f)] [7]. On the other hand, 


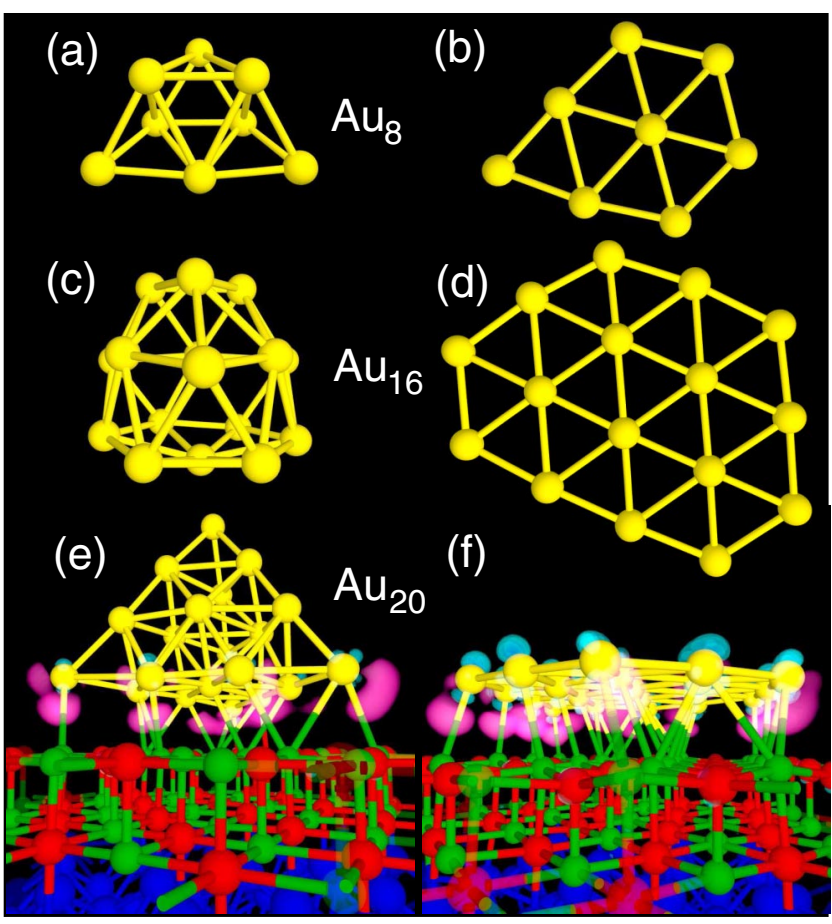

FIG. 2 (color online). Relaxed atomic configurations of isolated $\mathrm{Au}_{n}$ cluster isomers (a)-(d) and upon adsorption on the $\mathrm{MgO} / \mathrm{Mo}(100)$ (e)-(f). (a) $\mathrm{Au}_{8} 3 \mathrm{D}$ cluster isomer. (b) $\mathrm{Au}_{8}$ planar cluster isomer. (c) $\mathrm{Au}_{16}$ spherical-like cluster isomer. (d) $\mathrm{Au}_{16}$ planar cluster isomer. (e), (f) $\mathrm{Au}_{20}$ pyramidal (fcc) and planar cluster isomers, respectively. For the $\mathrm{Au}_{20}$ clusters (e) and (f) adsorbed on $\mathrm{MgO} / \mathrm{Mo}(100)$, we display the accumulated charge density at the cluster-oxide interface. Charge accumulation in pink and charge depletion in light blue. Yellow, green, red, and dark blue spheres correspond to $\mathrm{Au}, \mathrm{Mg}, \mathrm{O}$, and $\mathrm{Mo}$ atoms, respectively.

when adsorbed on the surface of $\mathrm{MgO} / \mathrm{Mo}(100)$ this $3 \mathrm{D}$ isomer becomes less stable than the planar one by $3.3 \mathrm{eV}$.

The adhesive energy gain found for $\mathrm{Au}_{n}$ adsorption on metal-supported thin $\mathrm{MgO}$ films originates from electronic charge redistribution effects induced by the presence of the metal substrate. In the following, we refer the electronic charge density of the combined systems, $\mathrm{Au}_{n} / \mathrm{MgO}$ or $\mathrm{Au}_{n} / \mathrm{MgO} / \mathrm{Mo}(100)$, to that of the isolated $\mathrm{Au}_{n}$ cluster and the support, $\mathrm{MgO}$ or $\mathrm{MgO} / \mathrm{Mo}(100)$. For this purpose we calculate the difference, $\triangle \rho(\vec{r})$, between the charge densities of the combined system and the separated components (cluster and support), with the latter calculated while keeping geometries as in the corresponding combined system. The redistributed charge is obtained through integration of $\triangle \rho(\vec{r})$ over the half-space containing the $\mathrm{Au}$ cluster, defined by a dividing plane parallel to the $\mathrm{MgO}$ surface and passing midway between the first two oxide layers. We find that the results are quite insensitive to variations of $\pm 0.5 \AA$ in the position of the dividing plane.

This analysis shows for $\mathrm{MgO}$ thin films supported on $\mathrm{Mo}(100)$ [with reference to $\mathrm{MgO}(100)$ ] accumulation of excess of charge [20] that has its origin in metal states that penetrate the thin oxide film. The difference between the integrated redistributed charge of the $\mathrm{Au}_{n} / \mathrm{MgO} / \mathrm{Mo}(100)$ and $\mathrm{Au}_{n} / \mathrm{MgO}$ systems yields the net amount of charge transferred from the metal to the cluster-oxide region [for $n=20$, see Figs. 2(e) and 2(f)]. In particular, for a given cluster size, the amount of charging increases with the contact area. For $\mathrm{Au}_{16}$ the amount of added charge due to the $\mathrm{Mo}(100)$ support goes from $0.3 e$ for a contact area comprised of $3 \mathrm{Au}$ atoms to $0.9 e$ for the planar isomer with $n_{\text {cont }}=16$. Similarly, for $\mathrm{Au}_{20}$ the additional charge provided by $\mathrm{Mo}(100)$ is $0.3 e$ for the pyramidal isomer while it increases up to about $1 e$ for the planar configurations with $n_{\text {cont }}=20$ [compare (e) and (f) in Fig. 2]. This trend is consistent with that exhibited by the energetic stability of the adsorbed Au clusters which increases with $n_{\text {cont }}$. The excess charge accumulated at the cluster-oxide interface leads to an electrostatic interaction with the Mo(100) substrate which gives rise to an energy gain that emerges as the dominant contribution to the adhesion of the gold clusters to $\mathrm{MgO}$. Both the overall enhancement of the cluster adhesion and the concomitant increase in stability of planar $\mathrm{Au}$ isomers are attributed to the electrostatic interaction which operates in the presence of the metal substrate [21].

The effectiveness of the electrostatic interaction discussed above depends on the thickness of the metalsupported $\mathrm{MgO}$ thin film. To illustrate this dependence we examine here the adsorption of $\mathrm{Au}_{8}$ clusters on the surface of $\mathrm{MgO} / \mathrm{Mo}(100)$, with the thickness of the oxide film varying from 2 to 7 layers. We find that regardless of the thickness of the oxide thin film, $\mathrm{Au}_{8}$ clusters in planar configurations $\left(n_{\text {cont }}=8\right)$ [Fig. 2(b)] are energetically favored with respect to $3 \mathrm{D}$ structures characterized by smaller $n_{\text {cont }}$ contact [Fig. 2(a)]. In particular, the difference between the adhesion energies of $\mathrm{Au}_{8}$ clusters adsorbed in the planar configuration and in the 3D structure decreases from $1.4 \mathrm{eV}$ to $0.8 \mathrm{eV}$ when the $\mathrm{MgO}$ film thickness increases from 2 to 7 layers. Furthermore, for $\mathrm{Au}_{8}$ clusters adsorbed on $\mathrm{MgO} / \mathrm{Mo}(100) \tilde{E}$ decreases from $0.7 \mathrm{eV}$ to $0.3 \mathrm{eV}$ as the thickness of the oxide film increases from 2 layers to 7 layers (Fig. 3). A similar dependence on thickness is recorded for the amount of charge transferred from the metal to the cluster-oxide interface (inset Fig. 3). The accumulated charge originating from the Mo(100) support decreases from $0.45 e$ to $0.1 e$ as the thickness of the film goes from 2 to 7 layers (Fig. 3), reflecting the decay of the metal states penetrating through the thin oxide films for thicker oxide films.

In summary, our first-principles investigations reveal that bonding, stability, energy distributions of isomeric adsorption configurations, and the charge states of gold nanoclusters deposited on thin $\mathrm{MgO}$ films are strongly influenced by the presence of a metal $[\mathrm{Mo}(100)]$ substrate supporting the thin MO film. On such films the adhesion energy of the Au clusters is significantly enhanced. This gain in adhesion correlates with the amount of electronic charge accumulated at the cluster-oxide interface, originating from metal states that penetrate through the $\mathrm{MgO}$ 


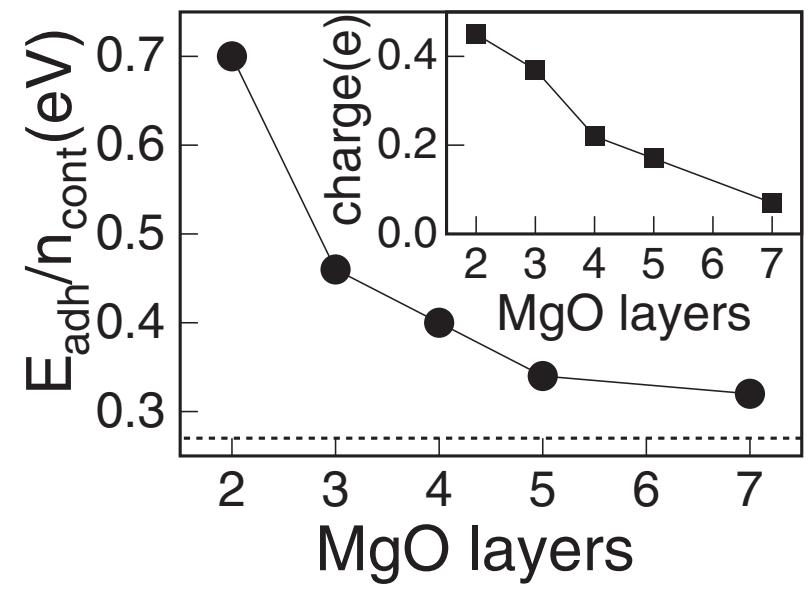

FIG. 3. Adhesion energy per contact atom $\left(n_{\text {cont }}=8\right), \tilde{E}$, of planar $\mathrm{Au}_{8}$ clusters adsorbed on $\mathrm{MgO} / \mathrm{Mo}(100)$ plotted vs the thickness (number of layers) of the $\mathrm{MgO}$ thin film. The inset shows the accumulated charge at the cluster-oxide interface plotted vs the number of $\mathrm{MgO}$ layers. The dashed line denotes $\tilde{E}$ for a planar $\mathrm{Au}_{8}$ cluster on a nondefective $\mathrm{MgO}(100)$ surface.

overlayers. The interfacial excess charge increases with the number of $\mathrm{Au}$ atoms in contact with the metal-oxide surface, and it underlies the emergence of an electrostatic adhesive energy term as a consequence of its interaction with the metal (Mo) support. This adhesive term is the origin of a dimensionality crossover from threedimensional to two-dimensional adsorbed cluster configurations, with the latter exhibiting maximal contact $\left(n_{\text {cont }}=\right.$ $n$ ) with the metal-oxide surface. The electrostatic effect decays with the $\mathrm{MgO}$ film thickness and remains appreciable up to $1 \mathrm{~nm}$ [22].

The dimensionality crossover induced by the metal support of the $\mathrm{MgO}$ film, the accompanying variations in the cluster electronic structure, and the added charging of the oxide-cluster interface are expected to influence physical and chemical properties of the adsorbed clusters, which can thus be controlled through the thickness of the $\mathrm{MgO}$ film. Since the above geometrical and electronic effects have been shown to be of paramount importance in catalytic processes involving metal-oxide supported gold nanoclusters [5,6,23], it is expected that our results will be employed in future investigations of the effect of underlying metal substrates on the nanocatalytic activity of such systems [24]. These investigations could provide the impetus for searching for alternative methods for controlling the interfacial charge (and consequently the chemical reactivity), for example, through the use of applied fields.

D. R., A. B., and U. L. acknowledge the support from the AFOSR and DOE. Computations were performed at the DOE National Energy Research Scientific Computing Center, at the Lawrence Berkeley National Laboratory
(NERSC) and at the Georgia Tech Center for Computational Material Science.

[1] U. Landman, Proc. Natl. Acad. Sci. U.S.A. 102, 6671 (2005).

[2] U. Heiz and U. Landman, Nanocatalysis (Springer, New York, 2006).

[3] M. Haruta, Gold Bulletin (London) 37, 27 (2004).

[4] M. Valden, X. Lai, and D. W. Goodman, Science 281, 1647 (1998).

[5] A. Sanchez et al., J. Phys. Chem. A 103, 9573 (1999).

[6] B. Yoon et al., Science 307, 403 (2005).

[7] L. M. Molina and B. Hammer, J. Catal. 233, 399 (2005).

[8] A. Bongiorno and U. Landman, Phys. Rev. Lett. 95, 106102 (2005).

[9] M.-C. Wu et al., Chem. Phys. Lett. 182, 472 (1991).

[10] G. Kresse and J. Hafner, Phys. Rev. B 47, R558 (1993).

[11] G. Kresse and J. Furthmuller, Phys. Rev. B 54, 11169 (1996).

[12] J. P. Perdew et al., Phys. Rev. B 46, 6671 (1992).

[13] D. Vanderbilt, Phys. Rev. B 41, 7892 (1990).

[14] L. Giordano, M. Baistrocchi, and G. Pacchioni, Phys. Rev. B 72, 115403 (2005).

[15] L. Giordano, J. Goniakowski, and G. Pacchioni, Phys. Rev. B 67, 045410 (2003).

[16] L. M. Molina and B. Hammer, Phys. Rev. B 69, 155424 (2004).

[17] S. Bulusu, Proc. Natl. Acad. Sci. U.S.A. 103, 8326 (2006).

[18] J. Li et al., Science 299, 864 (2003).

[19] H. Hakkinen et al., J. Phys. Chem. A 107, 6168 (2003).

[20] G. Pacchioni, L. Giordano, and M. Baistrocchi, Phys. Rev. Lett. 94, 226104 (2005).

[21] The interfacial electrostatic interaction can be described using the continuum model proposed by A. M. Stonheam and P. W. Tasker, Philos. Mag. B 55, 237 (1987); see Eqs. (2) and (3), with $\varepsilon_{\mathrm{MgO}}=9.8$ and $\varepsilon_{\mathrm{Mo}}=100$. Positioning the excess charge $1 \AA$ away from the oxide surface, and using for the charges corresponding to different number of $\mathrm{MgO}$ layers the values given in the inset of Fig. 3, yields for $E_{\text {adh }} / n_{\text {cont }}$ results that approximate well those shown in Fig. 3.

[22] A further increase in cluster adhesion may occur in the presence of oxygen vacancies ( $F$ centers) $[5,6]$. Indeed for an optimal 2D $\mathrm{Au}_{20}$ cluster, adsorbed on a 2-layer $\mathrm{MgO}$ film supported on $\mathrm{Mo}(100)$, with an oxygen vacancy in the top $\mathrm{MgO}$ layer, we find a $10 \%$ increase in $\tilde{E}$.

[23] H. Hakkinen et al., Angew. Chem., Int. Ed. 42, 1297 (2003).

[24] Preliminary calculations on the adsorption of $\mathrm{O}_{2}$ to $\mathrm{Au}_{8}$ anchored in the planar configuration on $\mathrm{MgO}(2 \mathrm{~L}) /$ Mo(100) show that the binding energy of the molecule at the periphery of the adsorbed cluster increases by about $0.8 \mathrm{eV}$ in comparison with the binding energy at the periphery of the 3D optimal $\mathrm{Au}_{8}$ structure on $\mathrm{MgO}(100)$. Furthermore, in the case of the metal-supported $\mathrm{MgO}$ film the adsorbed $\mathrm{O}_{2}$ molecule is highly activated in comparison to weak activation on (undefective) $\mathrm{MgO}(100)$ [6]. 